\title{
Seletividade de associações herbicidas pós-emergentes em variedade de soja precoce
}

\author{
Selectivity of postemergence herbicide associations in early soybean
}

\author{
Alberto Leão de L. Barroso ${ }^{1}$, André K. Shimohiro ${ }^{2}$, Hugo de Almeida Dan ${ }^{3}$, Lilian G.de M. \\ Dan $^{3}$, Joaquim J. de Almeida Júnior ${ }^{1}$, Sérgio de Oliveira Procópio ${ }^{4}$, Guilherme Braga P. Braz ${ }^{3}$
}

\begin{abstract}
Resumo - A frequente utilização de associações entre herbicidas pós-emergentes tem deixado inúmeras indagações a respeito da seletividade destes para a cultura da soja. Dessa maneira, o presente trabalho teve por objetivo avaliar a seletividade de misturas herbicidas em pósemergência na cultivar de soja de ciclo precoce Msoy $6101^{\circledR}$, levando em consideração a influência destas associações herbicidas sobre os caracteres agronômicos e produtividade desta variedade. O experimento foi realizado no município de Montividiu - GO, durante a safra 2004/2005, utilizando-se o delineamento experimental de blocos ao acaso, com quatro repetições, fazendo adoção de testemunhas duplas adjacentes. Os tratamentos testados foram: flumicloracpentil + chlorimuron-ethyl $\left(40,0+12,5 \mathrm{~g} \mathrm{ha}^{-1}\right)$, lactofen + chlorimuron-ethyl $\left(96,0+12,5 \mathrm{~g} \mathrm{ha}^{-1}\right)$, chlorimuron-ethyl + imazethapyr $\left(60,0+12,5 \mathrm{~g} \mathrm{ha}^{-1}\right)$, flumiclorac-pentil + chlorimuron-ethyl + clethodim $\left(40,0+12,5+60,0 \mathrm{~g} \mathrm{ha}^{-1}\right)$ e lactofen + chlorimuron-ethyl + haloxyfop-methyl $(96,0+$ $\left.12,5+42,0 \mathrm{~g} \mathrm{ha}^{-1}\right)$. Os resultados obtidos permitiram constatar que a associação entre os herbicidas lactofen + chlorimuron-methyl + haloxyfop-methyl causou níveis mais elevados de fitointoxicação, havendo redução na altura das plantas. Nenhuma das associações herbicidas reduziu o número de vagens por planta. Dentre todos os tratamentos, apenas a associação dos herbicidas flumiclorac-pentil + chlorimuron-ethyl + clethodim causou redução no ciclo e na produtividade da cultivar de soja precoce Msoy $6101^{\circledR}$.
\end{abstract}

Palavras-chave: Glycine max, precocidade, fitointoxicação.

\begin{abstract}
The frequent use of associations among postemergence herbicides has raised many questions about the selectivity of these for soybean crop. The objective of this work was to evaluate the selectivity of postemergence herbicide associations, and their influence on agronomic aspects, and yield of Msoy $6101^{\circledR}$, a soybean cultivar with early cycle. The study was conducted in Motevidiu - GO, during the 2004-2005 crop season. The experimental block design was entirely randomized in split-plot scheme with twofold checks, and four replicates. Tested treatments consisted of : flumiclorac - pentil + chlorimuron ethyl $\left(40+12,5 \mathrm{~g} \mathrm{ha}^{-1}\right)$, lactofen + chlorimuron ethyl $\left(96+12,5 \mathrm{~g} \mathrm{ha}^{-1}\right)$, chlorimuron ethyl + Imazethapyr $\left(60+12,5 \mathrm{~g} \mathrm{ha}^{-1}\right)$, flumiclorac - pentil + chlorimuron ethyl + clethodim $\left(40+12,5+60 \mathrm{~g} \mathrm{ha}^{-1}\right)$, and Lactofen + chlorimuron ethyl + haloxyfop methyl $\left(96+12,5+42 \mathrm{~g} \mathrm{ha}^{-1}\right)$. Results showed that the mixture lactofen + chlorimuron methyl + haloxyfop methyl caused the highest levels of phytotoxicity,
\end{abstract}

\footnotetext{
${ }^{1}$ Eng. ${ }^{\circ}$ Agr. Docente, Depto. Fitotecnia, FESURV, CEP: 75901-970, Rio Verde-GO, e-mail: all_barroso@hotmail.com

${ }^{2}$ Eng. ${ }^{\circ}$ Agr. Milenia agrociencias, Londrina, PR.

${ }^{3}$ Discentes do Programa de Pós Graduação em Agronomia pela Universidade Estadual de Maringá, Maringá, PR.

${ }^{4}$ Pesquisador, Embrapa Tabuleiros Costeiros, 49025-040, Aracaju, SE.
}

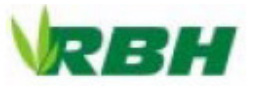


reducing plant height. No herbicide association reduced the number of pods per plant. However, the mixture flumiclorac-pentil + chlorimuron-ethyl + clethodin caused significant reduction on yield, and cycle of the early soybean cultivar Msoy $6101^{\circledR}$.

Key-words: Glycine max, early cycle, phytotoxicity.

\section{Introdução}

Nas últimas décadas, os programas de melhoramento genético tem contribuído enormemente para um aumento nas áreas de cultivo e produtividade da cultura da soja. Dentre os fatores que colaboraram para a elevação nas áreas de cultivo desta cultura destaca-se a criação de genótipos com ciclo mais precoce, o que viabilizou o cultivo em regiões onde existiam limitações climáticas para o desenvolvimento da soja (Lima et al., 2008).

A utilização de cultivares de soja de ciclo precoce tem diversos benefícios para o sistema agrícola, tais como: menor exposição ao ataque de pragas e patógenos (Hamawaki et al., 2002), menor gasto com defensivos e possibilidade da realização de safrinha. No entanto, por estas cultivares apresentarem período juvenil mais curto, a recuperação de condições de estresses é mais lenta.

A utilização de herbicidas na soja é frequente e a aplicação destes em pósemergência da cultura pode causar sintomas de fitointoxicação, sendo que estes sintomas variam de acordo com o mecanismo de ação de cada herbicida (Vargas et al., 2004). Hess (1995) cita que os sintomas de fitointoxicação causados pelos herbicidas inibidores da protoporfirinogênio oxidase caracterizam-se pela necrose apresentada nas folhas. Já os herbicidas inibidores da enzima ALS podem causar clorose, que pode ou não evoluir para necrose (Roman et al., 2007).

Para evitar que haja a fitointoxicação das plantas de interesse agrícola, é necessário estudar a seletividade destes produtos isolados e em associações com outros herbicidas. Em trabalhos realizados por Souza et al. (2002) e
Oliveira Jr. et al. (2006), não foram verificadas reduções de produtividade em função da aplicação de associações herbicidas pósemergentes na soja. Nelson et al. (2007) explicam que a seletividade expressa pelas plantas varia em função da dose e época de aplicação do herbicida.

Com relação às associações de herbicidas, Barros et al. (2000) não verificaram diferenças na altura das plantas entre os tratamentos herbicidas (chlorimuron-ethyl, lactofen, fomesafen, imazethapyr, chlorimuronethyl + lactofen, chlorimuron-ethyl + fomesafen, chlorimuron-ethyl + imazethapyr, imazethapyr + lactofen, imazethapyr + fomesafen) em relação à testemunha capinada. Em contrapartida, Hart et al. (1998) verificaram redução na produtividade da soja quando se utilizou a mistura entre thifensulfuron e bentazon em pós-emergência da cultura.

Diante da grande carência de trabalhos que avaliam o impacto das associações entre os herbicidas pós-emergentes e os seus efeitos sobre o desenvolvimento e produtividade de soja, o objetivo do presente trabalho foi avaliar a seletividade de associações herbicidas pósemergentes e a sua influência sobre os caracteres agronômicos e produtividade de uma cultivar de soja com ciclo precoce.

\section{Material e métodos}

O experimento foi conduzido na fazenda Pindaíbas, localizada no município de Montividiu-GO, cujas coordenadas geográficas são: latitude de $17^{\circ} 31^{\prime} 5.60^{\prime}$ ' S, longitude de $51^{\circ} 12^{\prime} 56.45^{\prime \prime}$ e altitude de 850 metros. O experimento foi realizado durante a safra de 2004/2005. 
O delineamento experimental utilizado foi o de blocos ao acaso, com quatro repetições, fazendo adoção de testemunhas duplas, onde para cada parcela com tratamento herbicida existem duas parcelas adjacentes sem a aplicação deste, de acordo com a metodologia descrita por Fagliari et al. (2001).

Cada unidade experimental possuía uma área útil de $7 \mathrm{~m}^{2}$. Os tratamentos herbicidas adotados foram: 1 - flumicloracpentil + chlorimuron-ethyl + adjuvante Lanzar ${ }^{\circledR}$ $0,2 \% \mathrm{v} \mathrm{v}^{-1}\left(40,0+12,5\right.$ g i.a. ha $\left.{ }^{-1}\right) ; 2$ - lactofen + chlorimuron-ethyl $\left(96,0+12,5\right.$ g i.a. ha $\left.{ }^{-1}\right) ; 3$ - chlorimuron-ethyl + imazethapyr $(12,5+60,0$ g i.a. ha $\left.{ }^{-1}\right) ; 4$ - flumiclorac-pentil + chlorimuron-ethyl + clethodim + adjuvante Lanzar $^{\circledR} 0,5 \% \mathrm{v} \mathrm{v}^{-1}(40,0+12,5+60,0$ g i.a. ha $\left.^{-1}\right) ; 5$ - lactofen + chlorimuron-ethyl + haloxyfop-methyl + adjuvante Assist ${ }^{\circledR} 0,5 \% \mathrm{v}$ $\mathrm{v}^{-1}\left(96,0+12,5+42,0\right.$ g i.a. ha $\left.{ }^{-1}\right)$.

A semeadura da soja foi realizada mecanicamente, com espaçamento entre linhas de 0,50 metros e uma densidade de 14 plantas por metro linear, totalizando uma população final de 280.000 plantas ha ${ }^{-1}$. A variedade de soja semeada foi a Monsoy $6101^{\circledR}$, que se caracteriza por apresentar ciclo precoce. Antecedendo a semeadura, foi realizada uma adubação a lanço, na qual se aplicou $80 \mathrm{~kg}$ de $\mathrm{P}_{2} \mathrm{O}_{5}$ ha $^{-1}+120 \mathrm{~kg}$ de $\mathrm{K}_{2} \mathrm{O}$ ha $^{-1}$. As sementes utilizadas na implantação do experimento foram tratadas com a mistura comercial à base dos fungicidas carboxin + thiram $(50 \mathrm{~g}$ de i.a. por $100 \mathrm{~kg}$ de sementes) e posteriormente procedeu-se a inoculação das mesmas.

No momento em que as plantas de soja encontravam-se no estádio fenológico V3, foi realizada a aplicação dos tratamentos herbicidas, utilizando-se um pulverizador costal com pressurização por $\mathrm{CO}_{2}$, com seis pontas de pulverização do tipo leque XR11002. Foi aplicado volume de calda equivalente a $200 \mathrm{~L} \mathrm{ha}^{-1}$. As condições climáticas no momento da aplicação eram favoráveis para que não houvesse perdas por deriva. Todas as unidades experimentais foram mantidas livres da presença de plantas daninhas com o objetivo de eliminar o efeito da matocompetição sobre a produtividade da soja.

Foram realizadas quatro avaliações visuais dos sintomas de toxicidade provocados pelos herbicidas nas plantas de soja, adotandose escala percentual de 0 (zero) a $100 \%$, em que zero representa ausência de sintomas e $100 \%$ morte de todas as plantas, aos 5, 18, 22 e 42 dias após a aplicação (D.A.A.). Na ocasião da colheita de cada tratamento, foram mensuradas a altura das plantas, altura de inserção da primeira vagem, número de vagens por planta e a duração do ciclo da soja.

A avaliação da produtividade foi realizada através da colheita da área útil de cada parcela, sendo os valores corrigidos para $13 \%$ de umidade. Os dados foram submetidos à análise de variância pelo teste $\mathrm{F}$ e as médias significativas comparadas pelo teste de Tukey, a $5 \%$ de probabilidade.

\section{Resultados e discussão}

Todos os tratamentos herbicidas causaram fitotoxicidade às plantas de soja, aos 5 DAA. (Tabela 1). Em geral, os sintomas nas plantas provocados pela aplicação dos herbicidas foram: redução de porte, encarquilhamento, clorose, necrose. Além destes sintomas, no tratamento chlorimuronethyl + imazethapyr foi verificado leve clorose internerval nas folhas.

Os herbicidas lactofen + chlorimuronethyl + haloxyfop-methyl provocaram níveis de toxicidade mais elevados dentre todos os tratamentos testados $(51,25 \%)$. Oliveira Jr. et al. (2006) verificaram sintomas semelhantes com relação à toxicidade provocada pelos herbicidas flumiclorac-pentil e lactofen aplicados em soja. 
Tabela 1. Valores médios de fitotoxicidade dos tratamentos herbicidas sobre a cultivar de soja Msoy $6101^{\circledR}$.

\begin{tabular}{lccccc}
\hline \multicolumn{1}{c}{ Tratamentos } & Dose & \multicolumn{4}{c}{ Dias após a aplicação } \\
\cline { 3 - 6 } & $\left(\mathbf{g}\right.$ i.a. ha $\mathbf{~}^{\mathbf{1}}$ ) & $\mathbf{0 5}$ & $\mathbf{1 8}$ & $\mathbf{2 2}$ & $\mathbf{4 2}$ \\
\hline Tlumic. + chlori. & $40,0+12,5$ & 27,50 & 11,25 & 8,75 & 0,00 \\
lacto. + chlori. & $96,0+12,5$ & 32,50 & 12,50 & 11,25 & 3,75 \\
chlori.+ imaze. & $60,0+12,5$ & 27,50 & 10,00 & 5,00 & 2,50 \\
flumic. + chlori. + cletho. & $40,0+12,5+60,0$ & 31,25 & 18,75 & 20,00 & 5,00 \\
lacto. + chlori. + haloxy. & $96,0+12,5+42,0$ & 51,25 & 26,25 & 31,25 & 18,75 \\
\hline lamic. (flumiclorac-pentil); chlori. (chlorimuron-ethyl); lacto. (lactofen); imaze. (imazethapyr); cletho. (clethodim);
\end{tabular}

Aos 18 DAA., houve redução média entre todos os tratamentos de $15,75 \%$ nas notas de fitointoxicação em relação à primeira avaliação. Assim como na primeira avaliação, os sintomas mais severos de toxicidade foram observados no tratamento com associação entre lactofen + chlorimuron-ethyl + haloxyfopmethyl, sendo que esta elevada toxicidade pode ser explicada em função da adição de adjuvante à calda de aplicação, pois em geral estes produtos potencializam a ação dos herbicidas de contato (Franzen et al., 2004). Espinosa et al. (1995) verificaram o mesmo potencial de elevação nos danos do lactofen, quando associado a um graminicida com adição de um óleo mineral, em comparação com lactofen isolado, corroborando resultados encontrados no presente ensaio.

Aos 22 DAA., a tríplice mistura lactofen + chlorimuron-methyl + haloxyfopmethyl manteve os maiores níveis de fitointoxicação $(31,2 \%)$, seguida do tratamento flumiclorac-pentil + chlorimuron-ethyl + clethodim, que apresentou 22,0\% (Tabela 1).

$\mathrm{Na}$ última avaliação (42 DAA.), os níveis de toxidez foram reduzidos em todos os tratamentos avaliados, chegando a valores aceitáveis, excluindo o tratamento com os herbicidas lactofen + chlorimuron-methyl + haloxyfop-methyl, que manteve elevado percentual de fitointoxicação $(18,0 \%)$. Verifica-se com o decorrer das avaliações que, mesmo a variedade empregada sendo de ciclo precoce, as plantas de soja demonstram excelente capacidade de recuperação dos sintomas provocados pelos herbicidas. Estes resultados corroboram os obtidos por Souza et al. (2002) e Oliveira Jr. et al. (2006).

$\mathrm{Na}$ avaliação de altura de plantas, o único tratamento que promoveu redução desta variável foi a tríplice mistura com os herbicidas lactofen + chlorimuron-methyl + haloxyfopmethyl, mostrando que esses herbicidas associados podem retardar o desenvolvimento desta cultura (Tabela 02). Resultados estes que estão de acordo com os encontrados por Souza et al. (2002), que verificaram que a mistura entre lactofen + chlorimuron-methyl causou reduções na altura das plantas de soja. Já Oliveira Jr. et al. (2006) concluiu que a utilização de flumiclorac-pentil ou lactofen, de forma isolada, não influencia a altura final das plantas de soja.

Analisando o número de vagens por plantas, verificou-se que as injúrias causadas pela aplicação dos herbicidas não foram capazes de alterar esta variável (Tabela 3). 
Tabela 2. Valores médios de altura de plantas dos tratamentos herbicidas sobre a cultivar de soja Msoy $6101^{\circledR}$.

\begin{tabular}{lccccc}
\hline \multicolumn{1}{c}{ Tratamentos } & Dose & \multicolumn{4}{c}{ Altura de plantas (m) } \\
\cline { 3 - 6 } & $\left(\mathbf{g}\right.$ i.a. ha $\mathbf{~ h}^{-\mathbf{1}}$ ) & \multicolumn{2}{c}{ Trat $^{1}$} & TD $^{2}$ & \\
\hline flumic. + chlori. & $40,0+12,5$ & 0,91 & $\mathrm{a}$ & 0,92 & $\mathrm{a}$ \\
lacto. + chlori. & $96,0+12,5$ & 0,83 & $\mathrm{a}$ & 0,93 & $\mathrm{a}$ \\
chlori.+ imaze. & $60,0+12,5$ & 0,92 & $\mathrm{a}$ & 0,92 & $\mathrm{a}$ \\
flumic. + chlori. + cletho. & $40,0+12,5+60,0$ & 0,92 & $\mathrm{a}$ & 0,91 & $\mathrm{a}$ \\
lacto. + chlori. + haloxy. & $96,0+12,5+42,0$ & 0,87 & $\mathrm{~b}$ & 0,92 & $\mathrm{a}$ \\
\hline CV\% & & 9,3 & 10,9 & \\
\hline
\end{tabular}

${ }^{1}$ Trat (tratamento); ${ }^{2} \mathrm{TD}$ (Testemunha dupla); flumic. (flumiclorac-pentil); chlori. (chlorimuron-ethyl); lacto. (lactofen); imaze. (imazethapyr); cletho. (clethodim); haloxy. (haloxyfop-methyl); médias seguidas de mesma letra na mesma linha não diferem entre si pelo teste de Tukey (5\% de probabilidade).

Este fato demonstra a capacidade que a cultura possui de se recuperar eficientemente dos sintomas de toxicidade, mesmo possuindo um ciclo de desenvolvimento mais precoce em comparação com outras variedades.

Avaliou-se, também, a altura de inserção da primeira vagem, que apresentou altura média de $0,13 \mathrm{~m}$ nas plantas de soja, e

Tabela 3. Valores médios do número de vagens por plantas no final do ciclo da cultivar de soja Msoy $6101^{\circledR}$.

\begin{tabular}{|c|c|c|c|c|c|}
\hline \multirow{2}{*}{ Tratamentos } & \multirow{2}{*}{$\begin{array}{c}\text { Dose } \\
\left(\mathrm{g} \text { i.a. } \text { ha }^{-1}\right)\end{array}$} & \multicolumn{4}{|c|}{ N.V.P.P. } \\
\hline & & Trat $^{1}$ & & $\overline{\mathrm{TD}}$ & \\
\hline flumic. + chlori. & $40,0+12,5$ & 15,28 & $\mathrm{a}$ & 14,21 & $\mathrm{a}$ \\
\hline lacto. + chlori. & $96,0+12,5$ & 14,48 & $\mathrm{a}$ & 14,89 & $\mathrm{a}$ \\
\hline chlori.+ imaze. & $60,0+12,5$ & 15,08 & $\mathrm{a}$ & 14,80 & $\mathrm{a}$ \\
\hline flumic. + chlori. + cletho. & $40,0+12,5+60$ & 14,68 & $\mathrm{a}$ & 14,64 & $\mathrm{a}$ \\
\hline lacto. + chlori. + haloxy. & $96,0+12,5+42,0$ & 14,68 & a & 14,35 & $\mathrm{a}$ \\
\hline $\mathrm{CV} \%$ & & 9,3 & & 10,9 & \\
\hline
\end{tabular}

* Número de vagens por planta; ${ }^{1}$ Trat (tratamento); ${ }^{2} \mathrm{TD}$ (Testemunha dupla); flumic. (flumiclorac-pentil); chlori. (chlorimuron-ethyl); lacto. (lactofen); imaze. (imazethapyr); cletho. (clethodim); haloxy. (haloxyfop-methyl); médias seguidas de mesma letra na mesma linha não diferem entre si pelo teste de Tukey (5\% de probabilidade).

Outra variável avaliada foi a duração do ciclo da soja, sendo esta influenciada em função dos diferentes tratamentos herbicidas utilizados (Tabela 4). Nas parcelas que receberam a aplicação da tríplice mistura flumiclorac-pentil + chlorimuron-ethyl + clethodim, as plantas atingiram a maturidade fisiológica com 101,5 dias, sendo o único não foram observadas diferenças entre os tratamentos e suas testemunhas adjacentes. Essa informação é de grande relevância para o processo de colheita mecanizada, pois uma redução na altura de inserção de primeira vagem pode causar perdas durante essa operação. 
$\mathrm{Na}$ avaliação de rendimento, apenas o tratamento com aplicação de flumiclorac-pentil + chlorimuron-ethyl + clethodim teve redução na produtividade quando comparado à sua testemunha adjacente, apresentando produtividade média de $2.916,2 \mathrm{~kg} \mathrm{ha}^{-1}$, sendo $11,2 \%$ menor que a sua testemunha (Tabela 4). Ressalta-se que essa redução de rendimento ocorreu em um tratamento que apresentou níveis aceitáveis de fitointoxicação na última avaliação realizada. Velini et al. (1992) explicam que a seletividade não deve ser determinada apenas pela verificação ou não de sintomas de toxicidade, pois são conhecidos os exemplos de herbicidas que podem reduzir a produtividade das culturas sem produzir efeitos visualmente detectáveis.
Mesmo sendo uma cultivar de ciclo precoce, a Msoy $6101^{\circledR}$ mostrou elevada capacidade de recuperação dos sintomas cloróticos e necróticos devido à aplicação da tríplice mistura lactofen + chlorimuron-methyl + haloxyfop-methyl, não tendo sua produtividade afetada. Estes dados corroboram os resultados obtidos por Peressin et al., (1997), que verificaram que a associação dos mesmos herbicidas, independente da adição de óleo mineral à calda, não causou quebra de produtividade da cultivar de soja IAC-8. Nelson et al. (2007) citam que o herbicida lactofen, até mesmo isolado, tem capacidade de reduzir a produtividade da soja, entretanto, para que isso ocorra, as plantas precisam apresentarse em condições de estresses.

Tabela 4. Valores médios de produtividade $\left(\mathrm{kg} \mathrm{ha}^{-1}\right)$ e ciclo da cultura (dias) dos tratamentos herbicidas sobre a cultivar de soja Msoy $6101^{\circledR}$.

\begin{tabular}{|c|c|c|c|c|c|}
\hline \multirow[t]{2}{*}{ Tratamentos } & \multirow{2}{*}{$\begin{array}{c}\text { Dose } \\
\left(\mathrm{g} \text { i.a. } \text { ha }^{-1}\right)\end{array}$} & \multirow{2}{*}{$\begin{array}{c}\text { Ciclo } \\
\text { (Dias) }\end{array}$} & \multicolumn{3}{|c|}{ Prod. $\left(\mathrm{kg} \mathrm{ha}^{-1}\right)$} \\
\hline & & & Trat $^{1}$ & $\mathbf{T D}$ & \\
\hline flumic. + chlori. & $40,0+12,5$ & 103,00 & a 3149,9 & a 3228,6 & $\mathrm{a}$ \\
\hline lacto. + chlori. & $96,0+12,5$ & 102,75 & a 3255,5 & a 3180,0 & $\mathrm{a}$ \\
\hline chlori.+ imaze. & $60,0+12,5$ & 102,25 & a 2976,6 & a 3306,8 & $\mathrm{a}$ \\
\hline flumic. + chlori. + cletho. & $40,0+12,5+60,0$ & 101,50 & b 2916,2 & b 3379,8 & $\mathrm{a}$ \\
\hline lacto. + chlori. + haloxy. & $96,0+12,5+42,0$ & 105,00 & a 3525,5 & a 3331,1 & $\mathrm{a}$ \\
\hline $\mathrm{CV} \%$ & & 11,40 & 8,50 & 10,01 & \\
\hline
\end{tabular}

Maturidade fisiológica; ${ }^{1}$ Trat (tratamento); ${ }^{2} \mathrm{TD}$ (Testemunha dupla); flumic. (flumiclorac-pentil); chlori. (chlorimuron-ethyl); lacto. (lactofen); imaze. (imazethapyr); cletho. (clethodim); haloxy. (haloxyfop-methyl); médias seguidas de mesma letra na mesma linha não diferem entre si pelo teste de Tukey (5\% de probabilidade).

\section{Conclusões}

Os resultados observados nas condições em que este experimento foi desenvolvido permitiram constatar que a associação entre os herbicidas lactofen + chlorimuron-methyl + haloxyfop-methyl causou níveis mais elevados de fitointoxicação, havendo inclusive redução na altura das plantas. Nenhuma das associações herbicidas reduziu o número de vagens por planta. Apenas a associação dos herbicidas flumiclorac-pentil + chlorimuron- ethyl + clethodim causou redução no ciclo e na produtividade da cultivar de soja precoce Msoy $6101^{\circledR}$.

\section{Referências}

BARROS, A.C. et al. Avaliação de herbicidas no controle de plantas daninhas na cultura da soja. Planta Daninha, v.10, n.1, p.315-319, 1992.

BARROS, A.C. et al. Eficiência e seletividade do lactofen em mistura com 
outros latifolicidas, no controle de plantas daninhas na cultura da soja. Revista Brasileira de Herbicidas, v.1, n.1, p.79-84, 2000.

BARKER, M.A. et al. Effects of 2,4-DB on soybean (Glycine max). Weed Science, v.32, n.1, p.299-303, 1984.

ESPINOSA, N.C. et al. Tolerância de soja (Glycine max) ao herbicida lactofen. Revista Ceres, v.42, n.1, p.10-24, 1995.

FAGLIARI, J.R. et al. Métodos de avaliação da seletividade de herbicidas para a cultura da cana-de-açúcar (Saccharum spp.). Acta Scientiarum Agronomy, v.23, n.2, p.12291234, 2001.

FRANZEN, D. W. et al. Influence of certain postemergence broadleaf herbicides on soybean stressed from iron deficiency chlorosis. Agronomic Journal, v.23, n.3, p.1357-1363, 2004.

HART, S.E. et al. Soybean (Glycine max) response to thifensulfuron and bentazon combinations. Weed Technology, v.12, n.1, p.179-184, 1998.

HAMAWAKI, O.T. et al. Avaliação da qualidade fisiológica e sanitária de sementes de genótipos de soja do ciclo precoce/médio em Uberlândia, Minas Gerais. Fitopatologia Brasileira, v.27, n.1, p.201-205, 2002.

HESS, F. D. Mode of action of photosynthesis inhibitors. In: Herbicide action course. Purdue University: Indiana, 1995, 787p.

LIMA, W.F. et al. Interação genótipoambiente de soja convencional e transgênica resistente a glifosato, no Estado do Paraná. Pesquisa agropecuária Brasileira, v.43, n.6, p.729-736, 2008.

NELSON, K. A. et al. Effect of lactofen application timing on yield and isoflavone concentration in soybean seed. Agronomic Journal, v. 99, n.1, p.645-649, 2007.
OLIVEIRA JR., R.S. et al. Aplicações seqüenciais de flumiclorac-pentil para o controle de Euphorbia heterophylla na cultura da soja. Acta Scientiarum Agronomy, v.28, n.1, p.115-122, 2006.

SOUZA, R.T. et al. Seletividade de combinações de herbicidas latifolicidas com lactofen para a cultura de soja. Scientia Agrícola, v.59, n.1, p.99-106, 2002.

ROMAN, E. S. et al. Como funcionam os herbicidas: da biologia à aplicação. Passo Fundo: Gráfica Editora Berthier, 2007. 160p.

VARGAS, L. \& ROMAN, E. S. Manual de Manejo e Controle de Plantas Daninhas. Bento Gonçalves: Embrapa Uva e Vinho, 2004. 652p.

YOUNG, B.G. \& YOUNG, J.M. Influence of late season postemergence soybean (Glicine max). Weed Technology, v.13, n.4, p.354-360, 2000.

TAIZ, L. \& ZEIGER, E. Fisiologia vegetal. Tradução de E.R. Santarem et al. 3 ed. Porto Alegre: Artmed, 2004. 720p.

VELINI, E.D. et al. Avaliação dos efeitos do herbicida clomazone, aplicado em pósemergência inicial, sobre o crescimento e produtividade de soqueira de cana-de-açúcar (Saccharum officinarum cv. SP 71-1406). STAB Açúcar, Álcool e Subprodutos, v.10, n.3, p.13-16, 1992. 